\title{
Teaching Undergraduate Macroeconomics with the Taylor-Romer Model
}

Paul Turner

\begin{abstract}
This paper sets out a version of the Taylor-Romer model of short-run macroeconomic equilibrium which can be used for teaching undergraduate economics principles courses. The aim is to generate a model with the proven advantages of the $I S-L M$ framework but with a more realistic description of central bank behaviour. The paper then provides a dynamic analysis of longer-term adjustment using a phase diagram but without the need for a formal mathematical derivation.
\end{abstract}

\section{Introduction}

A good pedagogical diagrammatic device (PDD for short) is of immense value to the teacher. Or, to put it rather less formally, a picture can be worth a thousand words. There is no shortage of examples of this old adage in the economics profession. Good examples are the partial equilibrium demand-supply diagram and the indifference map in microeconomics or the IS-LM diagram in macroeconomics. Without these devices it would become extraordinarily difficult to teach principles courses. At the same time it is not hard to think of cases in which 'teaching the diagram' has taken over from teaching the economics. A good lecturer needs to be able to distinguish between these cases and to choose the teaching method which best illuminates the underlying material.

This article seeks to ask the question of what constitutes a good PDD in economics. The particular question considered is whether or not the IS-LM model has outlived its usefulness or, can it be modified so that it remains relevant despite the radical changes in the macroeconomic environment which have taken place since Hicks (1937) first introduced it? In addressing this question, the model considered is that 
put forward by Taylor (2000) and Romer (2000) which now appears to be gaining a foothold in economics teaching (cf. Guest (2003) for a more extensive discussion).

The plan of the paper is as follows. In section II, the qualities that make for a good PDD in economics are discussed in general terms. This is followed in section III by a discussion of the extent to which current teaching devices such as IS-LM or AD-AS match these qualities are assessed and their shortcomings are identified. Section IV then introduces a version of the Taylor-Romer model and argues that this ameliorates some of the problems of existing devices discussed in the previous section. In section $V$ the Taylor-Romer model is extended to deal with the issue of dynamic price adjustment. Section VI contains conclusions.

\section{What constitutes a 'good diagram' in economics?}

Three desirable qualities stand out for any diagrammatic device in economics: these are simplicity, accuracy and flexibility. Each of these will be considered in turn.

\section{Simplicity}

In this context simplicity is used to mean that the diagram in question should have a sufficiently transparent structure to be easily assimilated by students. It may seem an obvious point but the important factor in teaching is usually to enable students to absorb the underlying economic principles rather than to spend time and effort in understanding how the diagram works. Of course the extent to which a diagram becomes 'too complicated' is a matter of subjective opinion for both lecturer and student. However, one example which the author has experienced is the use of the Edgeworth-Bowley box diagram in teaching general equilibrium analysis. In this case the degree of complexity in representing two sets of indifference curves in the same plane along with the shift in axes for different consumers means that explanation of the economic theory can easily take second place to explanation of just how the diagram 'works'. This does not mean to say that complete transparency is always a virtue. The effort needed to assimilate a model is often of value in itself in deepening the student's understanding of the underlying economic problem. The difficulty lies in achieving the right balance between these factors.

\section{Accuracy}

Teaching devices are normally used to provide an exposition of theory or to demonstrate how economic policy impacts on the economy. Since they are necessarily abstractions they inevitably fail to match the real world exactly. The question is whether the degree of mismatch is trivial or whether it is fatal. A PDD which strays too far from either the theory or the real world becomes a liability 
rather than an asset to the teacher. Teachers also need to be wary of the trade-off between simplicity and accuracy. Excessively simplified models have the advantage of being sufficiently transparent to be easily assimilated but the cost, in terms of the mismatch with real world economic structures, can often outweigh the benefits.

\section{Flexibility}

A good diagram should be capable of doing more than explaining the one point that the teacher wishes to make at a particular time. It should be a tool for students to use in analysing unfamiliar situations and extending their knowledge into issues which have not been directly addressed by the teacher. It is not hard to think of examples of teaching devices which are relevant in explaining a single issue but which have limited applications outside that particular topic. An example here might be the expectations augmented Phillips curve diagram. This provides a very useful device for illustrating the dynamics of inflation but does not provide a framework for thinking about a general class of policy problems. Contrast this with the IS-LM diagram which not only illustrates the determination of macroeconomic equilibrium but facilitates the analysis of a wide range of policy problems.

\section{IS-LM and AD-AS as teaching devices}

Having discussed the properties of a good diagrammatic device it is now possible to examine how some of the devices used in economics principles teaching match up to these criteria. Consider first the IS-LM model. Although most teachers of economics consider this a simple model, it is often the case that students express the view that it is 'too complicated'1. This may be the result of a mismatch between the objectives of teachers and students. There is no doubt that IS-LM requires some work before it is assimilated and that students often resent that work. However, teachers are in a position to see the future benefits and moreover, believe that the work itself is of value in pushing students to consider issues such as the determination of consumption and investment expenditures, the operation of the money market, the possibilities of market failure due to the liquidity trap and a host of other issues. In other words, for many teachers, IS-LM is not just a device for teaching the impact of government economic policy but a broad framework for teaching virtually the whole of macroeconomics. On that basis the view of the author is that IS-LM has the 'right' degree of simplicity - it requires some work to be assimilated but not so much that the effort outweighs its value.

The other criterion on which IS-LM scores highly is in terms of its flexibility. Although it is traditionally taught as a fix-price system, it is relatively easy to adapt it to consider the impact of price flexibility. Moreover, it is straightforward to extend the framework to deal with stochastic disturbances (cf. Poole (1970) ) or to deal with 
open economy issues (cf. Mundell (1963) and Fleming (1962)). With considerable justification Dornbusch (1980) describes Mundell's models (and by implication the IS-LM model underlying them) as 'the Volkswagens of the field - easy to drive, reliable and sleek'.

Where the IS-LM model does fall down is in terms of its accuracy or the degree to which its underlying assumptions match the structure of modern real-world economies. In particular the assumption that monetary policy consists of the authorities setting a fixed value of the money stock begins to look distinctly odd in a world of inflation targeting and persistent positive rates of inflation. The question arises as to whether this flaw in the model is fatal or whether it can be modified to restore its relevance. The argument of this paper is strongly in favour of the latter option.

Next consider the $A D-A S$ model which has now supplanted IS-LM in many economics principles textbooks. This model scores highly in terms of simplicity. In particular, its superficial similarity to the partial equilibrium demand-supply model taught on microeconomics courses makes it easy for students to assimilate and as a result, many students feel more confident in using this model than they do with $I S-L M$. In terms of flexibility, it is comparable to IS-LM. It can straightforwardly be extended to deal with stochastic shocks and, with a little imagination, open-economy issues. However, where this model does fail badly is in terms of accuracy. Again the basic assumption concerning monetary policy is that the authorities fix the value of the money stock. This leads to the unattractive feature that the 'equilibrium' is one in which the price level has converged on a constant value - an obviously unrealistic result which usually leads to immediate protests from students and desperate attempts by the teacher to apologise for the model and to patch it up with appropriate ad-hoc modifications.

In fact there are deeper problems with the $A D-A S$ model than the assumption of a fixed money stock. Colander (1995) has pointed out that the model contains two contradictory accounts of aggregate supply. In deriving the aggregate demand curve a fixed price multiplier theory is assumed while in deriving the aggregate supply curve the underlying assumption is one in which supply expands to the point at which marginal cost equals marginal revenue. Explaining this conflict to students means that the apparent simplicity of the model evaporates, leaving a far more complex muddle in its place.

To summarise: the view of this author is that both $I S-L M$ and $A D-A S$ are flawed teaching devices. However, the benefits of $I S-L M$ outweigh its flaws making it a powerful pedagogical tool which can enhance both teaching and learning. In contrast, $A D-A S$ is probably beyond rescue. As a teaching device it creates the 
problem that the lectures ends up spending more time trying to patch-up the model to get some degree of consistency with the real world rather than using the model as tool to understand that world.

\section{The Taylor-Romer Model}

The previous section considered some of the problems with the traditional teaching devices used on macroeconomic principles courses and advanced the argument that $I S-L M$ provides a less problematic approach than AD-AS. However, the problem of how to deal with the unrealistic modelling of monetary policy remains. The most promising suggestion has been the replacement of the LM curve with a more realistic policy rule along the lines suggested by Taylor (2000) or Romer (2000). If this is done then a simple core model can be written in the form of equations (1) - the IS curve, (2) the monetary policy function and (3) the Phillips curve $^{2}$

$$
\begin{aligned}
& y=\alpha_{0}-\alpha_{1}\left(i-\pi^{e}\right)+\alpha_{2} g \\
& i=\bar{r}+\pi^{e}+\gamma_{1}(\pi-\bar{\pi})+\gamma_{2}(y-\bar{y}) \\
& \pi=\pi^{e}+\beta_{1}(y-\bar{y})
\end{aligned}
$$

where $y$ is real output, $i$ is the nominal interest rate, $\pi e$ is the expected rate of inflation, $g$ is government spending, $\bar{r}, \bar{\pi}$, and $\bar{y}$ are the central bank's targets for the real interest rate, inflation and output $\pi$ while is the actual rate of inflation. All coefficients are assumed to be positive. Note that it is assumed that the central bank's target level for output $\bar{y}$ is equal to the capacity level of output which appears in the Phillips curve.

Monetary policy in this extended framework has a much richer interpretation than in the simple IS-LM model. Here monetary policy involves the choice of targets for the real interest rate, output and inflation as well as the choice of how strongly to react when these targets are breached as reflected in the coefficients. Substituting (3) into (2) gives a modified monetary policy function of the form ${ }^{3}$ :

$$
i=\overline{r^{*}}+\left(1+\gamma_{2}\right) \pi^{e}+\left(\gamma_{1}+\gamma_{2} \beta_{1}\right)(y-\bar{y})-\gamma_{2} \bar{\pi}
$$

Given equation (4) it is now possible to construct a simple diagram analogous to the $I S-L M$ model in which the goods market is represented by the $I S$ curve (1) and the $L M$ representation of monetary policy is replaced by (4). To emphasise the distinction between the two models, the diagrammatic representation of equation (4) is labelled the MP (monetary policy) curve. Note however that monetary policy can now be interpreted in a much more realistic manner. Instead of the unrealistic 
Figure 1: Effects of a increase in the target rate of inflation

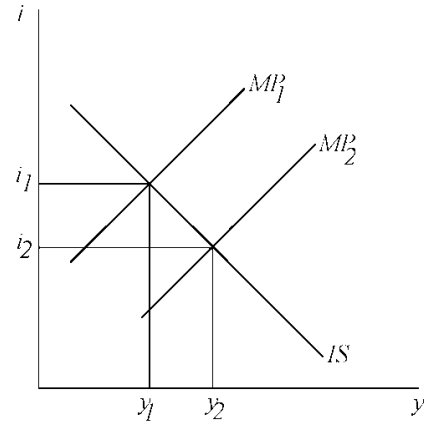

assumption that monetary policy involves simply fixing the money supply, it now reflects decisions as to the target rates of inflation and output and the extent to which the interest rate responds when these targets are breached.

Consider an expansionary monetary policy, which is here defined as an increase in the target rate of inflation. This is illustrated in Figure 1 by a shift to the right of the $M P$ curve resulting from a higher value of $\bar{\pi}$. In the new equilibrium the interest rate has fallen and output has increased.

The shift in the equilibrium illustrated in Figure 1 depends critically on the assumption that inflation expectations do not change when the change in inflation target is implemented. Consider instead an alternative scenario in which the change in inflation target is announced and the private sector immediately adjusts expectations. In terms of equation (4) we can replace both $\pi e$ and $\bar{\pi}$ with $\bar{\pi}_{2}$, the new target rate of inflation. We also need to adjust the expectations term in the IS curve (1) to allow for the change in policy. This produces a very different equilibrium as illustrated by Figure 2. Here the IS curve has shifted to the right to reflect the change in expectations while the MP curve has shifted to the left. In the new equilibrium output is unchanged and the nominal interest rate has fully adjusted to the new expected rate of inflation to leave the real interest rate unchanged.

In the construction of Figures 1 and 2, a general expression for the monetary policy function has been used, and this has produced an upward sloping MP curve. Existing papers advocating the replacement of the $L M$ curve with a monetary policy function have generally simplified the model by assuming a horizontal MP curve (cf. Romer (2000) for example). This allows the policy discussion to be conducted in terms of the central bank setting a fixed interest rate. However, this 
Figure 2: Effects of an anticipated increase in the target rate of inflation

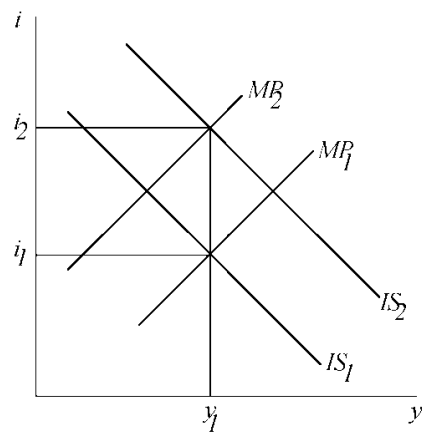

simplification comes at a price. In order to generate this result we need to make two potentially restrictive assumptions - first, we must assume that the central bank sets a weight of zero on deviations of output from target in its monetary policy rule i.e. $\gamma_{2}=0$ and second, we must assume that there is no contemporaneous effect of the output gap on inflation i.e. $\beta_{l}=0^{4}$. Given this, we prefer the less restricted model even if this slightly complicates the subsequent policy discussion.

\section{Dynamic Adjustment with the Taylor-Romer Model}

Discussions of dynamic adjustment are always problematic in undergraduate economics principles courses. The most natural 'technology' for such a discussion is the phase diagram. However, a formal mathematical derivation is often too demanding for students at undergraduate level and some alternative must be found. A possible half-way stage between a purely informal discussion and a full derivation of the time-path using differential or difference equations, is to discuss the adjustment mechanism(s) relatively loosely and then use the phase diagram as a descriptive tool.

For example, suppose the aim is to analyse the adjustment of the economy to a cut in the target rate of inflation. A starting point might be that expected or core inflation is 'sticky' and that it takes time to respond to changes in the actual rate of inflation. It is possible to use a phase diagram to illustrate the effects of a cut in the target rate of inflation as shown in Figure 3 which has the interest rate and output on the axes as in the conventional IS-LM diagram. The economy begins in equilibrium at point $A$ where the original $I S$ curve $I S_{1}$ intersects with a monetary policy function $M P_{1}$ consistent with the original target rate of inflation $\bar{\pi}_{1}$. The cut in 
Figure 3: Adjustment to a cut in the target rate of inflation

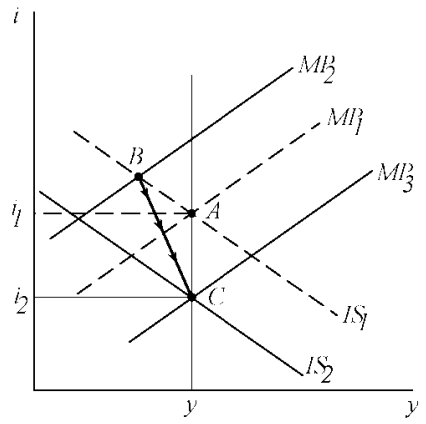

the target rate of inflation to $\bar{\pi}_{2}$ initially produces a shift to the left of the monetary policy function to $M P_{2}$ and the short-run equilibrium moves to point $B$ at which the interest rate has risen and output has fallen. However, at point $B$ inflation starts to fall and the monetary policy function starts to shift to the right while the IS curve shifts to the left as expected inflation starts to fall in line with actual inflation. This continues until a new equilibrium is established at point $C$ where output is at its capacity level and the nominal interest rate has fallen by the same amount as the cut in the inflation. The result is that the real interest rate has not changed relative to the original equilibrium. Note that the model with a Taylor rule does not suffer from potential instability as is the case with dynamic analysis of the standard IS-LM model with a Phillips curve (cf.Tobin (1975)).

\section{Conclusions}

This article has built on the insights of a growing literature concerning the best way of teaching macroeconomic principles at the undergraduate level. It has been argued that the Taylor-Romer model offers significant advantages in terms of its description of central bank behaviour and the realism of its underlying assumptions. The argument is that a comparative static diagram which constitutes an adaptation of the familiar IS-LM diagram provides an excellent way of illustrating short-term macroeconomic equilibrium. For longer-term adjustment processes, the recommended approach is to use a phase diagram but without the formal mathematical derivation of the underlying dynamic model. 


\section{References}

Colander, D. (1995) 'The Stories We Tell: A Reconsideration of AS/AD Analysis', Journal of Economic Perspectives, 9(2), pp.169-188.

Dornbusch, R. (1980) Open Economy Macroeconomic, New York: Basic Books.

Fleming, J. M. (1962) ‘Domestic Financial Policies under Fixed and under Floating Exchange Rates', IMF Staff Papers, 9(3) pp. 369-379.

Guest, R. (2003) 'Modifying the Taylor-Romer Model of Macroeconomic Stabilisation for Teaching Purposes', International Review of Economics Education, 2(1), pp. 58-68.

Hicks, J.R. (1937) 'Mr Keynes and the "Classics"; A Suggested Interpretation', Econometrica 5, pp. 147-159.

Mundell, R. A. (1963) 'Capital Mobility and Stabilization Policy under Fixed and Flexible Exchange Rates' Canadian Journal of Economics and Political Science, 29(4), pp. 487-499.

Poole, W. (1970), 'The optimal choice of monetary instrument in a simple stochastic macro model', Quarterly Journal of Economics, 84, pp. 197-221.

Romer, D., (2000) "Keynesian Macroeconomics without the LM Curve", Journal of Economic Perspectives, 14, 149-169.

Taylor, J. B.(2000) "Teaching Modern Macroeconomics at the Principles Level," American Economic Review, 90, 90-94.

Tobin, J. (1975) 'Keynesian Models of Recession and Depression' American Economic Review Papers and Proceedings, Volume 65, Issue 2, pp 195-202.

\section{Notes}

1 This is a largely subjective opinion but one based on twenty years of teaching principles courses. I suspect that most (if not all) teachers of undergraduate economics will have come across something similar. The problem is not so much with the completed diagram itself but with the process involved in building it up from an analysis of individual behavioural relationships.

2 The use of equations here should not be interpreted as a recommendation for their use in a mainstream principles course. Advanced students may enjoy this approach but the majority are usually more comfortable with a diagrammatic presentation.

3 A basic point (but one which is not often made) is that the actions of the fiscal and monetary authorities must be consistent. For the economy to reach an equilibrium we must have, $r=\frac{\alpha_{3}+\alpha_{2}, g-\bar{y}}{\alpha_{1}}$, that is the target real rate of interest must clear the goods market at capacity output given the level of government spending. How this consistency can be achieved is not immediately obvious but it will be assumed throughout that this is the case.

4 Note from equation (4) that $\frac{d}{d v}=\gamma_{2}+y_{1} \beta_{1}$ along the monetary policy function. It follows that, if $\gamma_{1}>0$ (which is required for stability), then this function has zero slope if and only if $\gamma_{1}=0$ and $\beta_{1}=0$.

\section{Acknowledgements}

Thanks are due to two anonymous referees for comments on an earlier version of this paper. 
International Review of Economics Education

\section{Contact details}

Dr Paul Turner

Department of Economics

Loughborough University

Loughborough

LE11 3TU

email: p.m.turner@lboro.ac.uk 